\title{
Fluoropyrimidine-Associated Toxicity in Two Gastrointestinal Cancer Patients: Potential Role of Common DPYD Polymorphisms
}

\author{
Felicia Stefania Falvella ${ }^{a}$ Marco Luoni $^{\mathrm{b}}$ Stefania Cheli ${ }^{\mathrm{a}}$ Sergio Fava ${ }^{\mathrm{b}}$ \\ Massimiliano Cergnul ${ }^{b}$ \\ a Unit of Clinical Pharmacology, Department of Laboratory Medicine, ASST Fatebenefratelli-Sacco, Milan, and \\ ${ }^{b}$ Department of Medical Oncology, Ospedale Civile di Legnano, ASST Ovest Milanese, Legnano, Italy
}

\section{Established Facts}

- Fluoropyrimidine-based therapy is used extensively in oncology for the treatment of several solid tumors including gastrointestinal types of cancer.

- Some patients experience fluoropyrimidine-associated toxicity. The frequency and severity of these adverse events vary from patient to patient and are partially due to genetic polymorphism into the dihydropyrimidine dehydrogenase (DPYD) gene.

\section{Novel Insights}

- $D P Y D^{*} 2 \mathrm{~A}, D P Y D^{*} 13$, or $D P Y D$ D949V has a clear impact on the reduced enzyme activity but can account only for a small percentage of the toxicity due to a low allelic frequency.

- Our cases seem to reveal an upfront reduction of the fluoropyrimidine starting dose in patients carrying DPYD V732I in the homozygous or compound heterozygous state, although the exact degree of dose reduction should be determined in larger patient populations.

\section{Keywords}

Fluoropyrimidine $\cdot$ Toxicity $\cdot D P Y D \cdot$ V732I

\section{Abstract}

While the majority of patients can be treated safely with fluoropyrimidine, some experience severe fluoropyrimidine-associated toxicity. The frequency and severity of these adverse events vary from patient to patient and are partially explained by genetic polymorphism into the dihydropyrimidine dehydrogenase $(D P Y D)$ gene. Carriers of the rare allelic variants $D P Y D^{*} 2 \mathrm{~A}, D P Y D^{*} 13$, and DPYD D949V are more likely to experience severe adverse reactions during fluoropyrimidine-based therapy. However, these 3 genetic variants explain only a small percentage of the overall drug toxicity, and more frequent ones such as homozygous or compound heterozygous DPYD V732I can play a key role.

(c) 2017 S. Karger AG, Basel

\section{KARGER}

(C) 2017 S. Karger AG, Basel

E-Mail karger@karger.com

www.karger.com/che
Felicia Stefania Falvella

Department of Laboratory Medicine, ASST Fatebenefratelli-Sacco

Via Giovanni Battista Grassi 74

IT-20157 Milan (Italy)

E-Mail stefania.falvella @asst-fbf-sacco.it 
Table 1. Genotyping analysis performed in our cases

\begin{tabular}{|c|c|c|c|c|c|c|}
\hline Variant & SNP ID & MAF, $\%$ & Variant effect & Reference $^{a}$ & Case 1 & Case 2 \\
\hline$D P Y D$ c. $496 A>G$ & rs2297595 & 12 & p.Met166Val & 3 & AA & AA \\
\hline$D P Y D$ c. $1129-5923 C>\mathrm{G}$ & rs75017182 & 2 & splice site & 9,10 & $\mathrm{CC}$ & $\mathrm{CG}^{\mathrm{b}}$ \\
\hline$D P Y D^{*} 13$ c. $1679 \mathrm{~T}>\mathrm{G}$ & rs55886062 & 0.1 & p.Ile560Ser & 1 & TT & $\mathrm{TT}$ \\
\hline$D P Y D^{*} 2 \mathrm{~A}$ c. $1905+1 \mathrm{G}>\mathrm{A}$ & rs3918290 & 0.5 & splice site & 1 & GG & GG \\
\hline$D P Y D^{*} 6$ c. $2194 \mathrm{G}>\mathrm{A}$ & rs 1801160 & 5 & p.Val732Ile & 4 & $\mathrm{AA}^{\mathrm{b}}$ & $\mathrm{GA}^{\mathrm{b}}$ \\
\hline$D P Y D$ c. $.2846 \mathrm{~A}>\mathrm{T}$ & rs67376798 & 0.7 & p.Asp949Val & 1 & AA & AA \\
\hline
\end{tabular}

DPYD variants are listed in order of the location on the gene. SNP, single-nucleotide polymorphism; MAF, minor allele frequency in the European population (www.ensembl.org). ${ }^{\text {a }}$ References reporting an association between $D P Y D$ variants and fluoropyrimidine toxicity. ${ }^{\mathrm{b}}$ Genotypes reported to be associated with toxicity.

\section{Introduction}

Fluoropyrimidine-based therapy is used extensively in oncology for the treatment of several solid tumors including gastrointestinal types of cancer. While the majority of patients can be treated safely, some experience severe fluoropyrimidine-associated toxicity, which may be lethal. The frequency and severity of these adverse events vary from patient to patient and are partially explained by genetic polymorphism into the dihydropyrimidine dehydrogenase gene (DPYD). This gene encodes for the initial and rate-limiting enzyme involved in the pyrimidine catabolism, i.e., dihydropyrimidine dehydrogenase (DPD), which converts 5-fluorouracil (5-FU) to dihydrofluorouracil (DHFU). Carriers of the allelic variants DPYD*2A (intronic), DPYD*13 (I560S), and rs67376798 (D949V) are more likely to experience severe adverse reactions during fluoropyrimidine-based therapy, and consequently the Clinical Pharmacogenetics Implementation Consortium Guidelines recommend an alternative drug for individuals with allelic variants with a homozygous status and a starting dose of $50 \%$ for heterozygous carriers [1]. Due to a low allelic frequency, however, these 3 variants explain only a small percentage of the overall drug toxicity and more frequent $D P Y D$ variants can play a key role in this undetected heritability $[2,3]$.

Recently, Boige et al. [4] made important advances in this direction by investigating $25 D P Y D$ variants in a large cohort of patients with stage III colon cancer treated with FOLFOX4 adjuvant chemotherapy. Among the 12 DPYD variants found to be polymorphic, statistically significant associations with grade 3 or greater fluorouracil adverse events were observed for carriers of rs67376798 (D949V: OR 6.3; 95\% CI 2.0-27.0) and rs1801160 (V732I: OR 1.7; 95\% CI 1.3-2.4) [4]. That study also confirmed the signif- icant predictive value of rs67376798 (D949V), but not for $D P Y D^{*} 2 \mathrm{~A}$ and $D P Y D^{*} 13$, which are the other variants included in the Clinical Pharmacogenetics Implementation Consortium Guidelines. This apparently surprising discrepancy may simply be due to the low allelic variant frequency in the population studied by Boige et al. [4]. Consequently, a strategy considering more frequent $D P Y D$ variants may be relevant to understand the cause-effect correlation between toxicity and heritability, which is currently lacking in the assessment of only rare variants. We investigated 2 cases of fluoropyrimidines-associated toxicity which were subsequently tested for DPYD functional polymorphisms to understand the origin of the toxicity.

\section{Case 1}

In December 2015, a 69-year-old woman underwent a right colectomy. During surgery peritoneal carcinomatosis was found and liver metastases emerged from the instrumental tests (final clinical stage IV). In February 2016 we started a first-line chemotherapy with FOLFOX-3 (oxaliplatin at $85 \mathrm{mg} / \mathrm{m}^{2}$ on day 1 and leucovorin at $500 \mathrm{mg} / \mathrm{m}^{2}$ and $5-\mathrm{FU}$ at $3,000 \mathrm{mg} / \mathrm{m}^{2}$ as a $46-\mathrm{h}$ infusion starting on day 1$)$ and bevacizumab $(5 \mathrm{mg} / \mathrm{kg}$ on day 1$)$ every 2 weeks, with a reduced dose (to 75\%) of oxaliplatin to for the kidney failure [5]. After the second cycle of chemotherapy grade 2 stomatitis appeared, while after the fourth cycle grade 4 diarrhea with significant dehydration and acute renal failure led to hospitalization in April 2016. In view of the toxicity, we continued folinic acid and 5-FU at dosages reduced to 75\%. During the course of further chemotherapy, the patient had no additional toxicity or any hematological or gastrointestinal issues.

\section{Case 2}

In July 2016 a pulmonary and hepatic recurrence of an ampulla Vater adenocarcinoma was diagnosed in a 69-year-old man. In August 2016 a first-line full-dose chemotherapy was commenced
Falvella/Luoni/Cheli/Fava/Cergnul 
following an mFLOX (modified FLOX) regimen (oxaliplatin at a dose of $85 \mathrm{mg} / \mathrm{m}^{2}$ on days 1 and 8 and leucovorin at a dose of 50 $\mathrm{mg} / \mathrm{m}^{2}$ and $5-\mathrm{FU}$ at a dose of $500 \mathrm{mg} / \mathrm{m}^{2}$ on days 1,8 , and 15 every 28 days). After the first cycle, the patient had grade 2 nausea and vomiting, loss of appetite, grade 2 diarrhea, deterioration of his general condition, and a weight loss of $8 \mathrm{~kg}$. The patient refused to continue with further chemotherapy due to toxicity.

\section{Genotyping}

The patients signed a written informed consent before genotyping. Genomic DNA was isolated from peripheral blood cells using an automatic DNA extraction system (Maxwell ${ }^{\circledR} 16$ System; Promega, Madison, WI, USA) according to the manufacturer's instructions. All genotypes were determined by real-time PCR using a panel of LightSNiP from TIB-MolBiol (Berlin, Germany). At the end of the amplification a melting curve analysis was performed (LightCycler 480; Roche, Mannheim, Germany). We evaluated the well-known functional variants that are associated with fluoropyrimidine toxicity (Table 1). A pharmacogenetic test of case 1 revealed the presence of V732I in the homozygous state, and in case 2 the heterozygous state for V732I and for the intronic variant c.1129-5923C $>$ G was found. This specific pharmacogenetic trait observed in both patients is compatible with areduced DPD activity and may help physicians decide whether to reduce the fluoropyrimidine dose (Table 1 ).

\section{Discussion}

Fluoropyrimidine-associated toxicity is a well-recognized clinical problem that impacts a patient's quality of life. The goal of pharmacogenetics is to develop a panel of functional variants that are able to define a reduced DPD enzyme activity. As rightly underlined by Deenen and Meulendijks [6], screening for DPYD variants may not eliminate all fluoropyrimidine-associated toxicities; however, the evidence regarding the clinical utility of $D P Y D$ genotype-guided dosing of fluoropyrimidines is such that it should be considered in international clinical practice guidelines. Until now, pretherapeutic DPYD pharmacogenetic testing to prevent fluoropyrimidine-related toxicities has not been a very common practice in medical oncology; however, the evidence of the clinical validity and specificity of the $D P Y D^{*} 2 \mathrm{~A}, D P Y D^{*} 13$, and $D P Y D$ rs67376798 genotyping test to prevent fluoropryrimidine-related toxicity and to preserve treatment compliance could change the habits in medical oncology [7, 8]. The more common deep intronic variant c.11295923C $>\mathrm{G}$ has been significantly linked to severe 5-FU toxicity $[9,10]$. This variant affects DPD pre-mRNA splicing by creating a cryptic splice donor site and results in a premature stop codon [9]. The effect of the new common nonsynonymous DPYD variant V732I is still not fully elucidated, and we cannot rule out that V732I may only be in linkage disequilibrium with the causal variants. Since its association with fluorouracil-related adverse events was found in a large study of stage III colon cancer patients treated with FOLFOX4 chemotherapy, its role could be crucial. Our report advocates for a more comprehensive genotyping approach to safely administer the fluoropyrimidine dose, with the goal of obtaining the highest chance of cure despite the risk of treatment-related toxicity. However, further studies are needed to confirm and quantitate the V732I associations before including it, as homozygous or compound heterozygous, into a pretherapeutic DPYD pharmacogenetic model of fluoropryrimidine-related toxicity.

\section{Disclosure Statement}

The authors have no conflicts of interest to declare.

\section{References}

1 Caudle KE, Thorn CF, Klein TE, Swen JJ, McLeod HL, Diasio RB, Schwab M: Clinical Pharmacogenetics Implementation Consortium guidelines for dihydropyrimidine dehydrogenase genotype and fluoropyrimidine dosing. Clin Pharmacol Ther 2013;94:640645.

2 Falvella FS, Caporale M, Cheli S, Martinetti A, Berenato R, Maggi C, Niger M, Ricchini F, Bossi I, Di Bartolomeo M, Sottotetti E, Bernardi FF, de Braud F, Clementi E, Pietrantonio F: Undetected toxicity risk in pharmacogenetic testing for dihydropyrimidine dehydrogenase. Int J Mol Sci 2015;16:8884-8895.
3 Falvella FS, Cheli S, Martinetti A, Mazzali C, Iacovelli R, Maggi C, Gariboldi M, Pierotti MA, Di Bartolomeo M, Sottotetti E, Mennitto R, Bossi I, de Braud F, Clementi E, Pietrantonio F: DPD and UGT1A1 deficiency in colorectal cancer patients receiving triplet chemotherapy with fluoropyrimidines, oxaliplatin and irinotecan. Br J Clin Pharmacol 2015;80:581-588.

4 Boige V, Vincent M, Alexandre P, Tejpar S, Landolfi S, Le Malicot K, Greil R, Cuyle PJ, Yilmaz M, Faroux R, Matzdorff A, Salazar R, Lepage C, Taieb J, Laurent-Puig P: DPYD genotyping to predict adverse events following treatment with flourouracil-based adjuvant chemotherapy in patients with stage III colon cancer: a secondary analysis of the PETACC-8 randomized clinical trial. JAMA Oncol 2015, Epub ahead of print.

5 Takimoto $\mathrm{CH}$, Graham MA, Lockwood G, Ng CM, Goetz A, Greenslade D, Remick SC, Sharma S, Mani S, Ramanathan RK, Synold TW, Doroshow JH, Hamilton A, Mulkerin DL, Ivy P, Egorin MJ, Grem JL: Oxaliplatin pharmacokinetics and pharmacodynamics in adult cancer patients with impaired renal function. Clin Cancer Res 2007;13:48324839 .

Fluoropyrimidine-Associated Toxicity

Chemotherapy 2017;62:323-326 DOI: $10.1159 / 000477333$ 
6 Deenen MJ, Meulendijks D: Recommendation on testing for dihydropyrimidine dehydrogenase deficiency in the ESMO consensus guidelines for the management of patients with metastatic colorectal cancer. Ann Oncol 2016;pii:mdw533.

7 Toffoli G, Giodini L, Buonadonna A, Berretta M, De Paoli A, Scalone S, Miolo G, Mini E, Nobili S, Lonardi S, Pella N, Lo Re G, Montico M, Roncato R, Dreussi E, Gagno S, Cecchin E: Clinical validity of a DPYD-based pharmacogenetic test to predict severe toxicity to fluoropyrimidines. Int J Cancer 2015;137:29712980.
8 Bermejo-Pérez MJ, Galeote-Miguel AM, Rodelo-Haad LE, Alés-Díaz I, Durán-Ogalla G, Benavides-Orgaz M: Toxicity associated with capecitabine in patients suffering from dihydropyrimidine dehydrogenase deficiency. Chemotherapy 2014;60:353-355.

9 van Kuilenburg AB, Meijer J, Mul AN, Meinsma R, Schmid V, Dobritzsch D, Hennekam RC, Mannens MM, Kiechle M, EtienneGrimaldi MC, Klümpen HJ, Maring JG, Derleyn VA, Maartense E, Milano G, Vijzelaar R, Gross E: Intragenic deletions and a deep intronic mutation affecting pre-mRNA splicing in the dihydropyrimidine dehydrogenase gene as novel mechanisms causing 5-fluorouracil toxicity. Hum Genet 2010;128:529-538.
10 Lee AM, Shi Q, Alberts SR, Sargent DJ, Sinicrope FA, Berenberg JL, Grothey A, Polite B, Chan E, Gill S, Kahlenberg MS, Nair SG, Shields AF, Goldberg RM, Diasio RB: Association between DPYD c.1129-5923 C>G/ hapB3 and severe toxicity to 5-fluorouracilbased chemotherapy in stage III colon cancer patients: NCCTG N0147 (Alliance). Pharmacogenet Genomics 2016;26:133-137. 